\title{
Publisher Correction: Universal gap scaling in percolation
}

Jingfang Fan (D), Jun Meng, Yang Liu, Abbas Ali Saberi, Jürgen Kurths and Jan Nagler (D)

Correction to: Nature Physics https://doi.org/10.1038/s41567-019-0783-2, published online 10 February 2020.

In the version of this Article originally published online, in Fig. $1 \mathrm{~d}$ and its caption and in Fig. 3d, in all instances of ' $\chi_{r}$ ' subscript ' $r$ ' should have been ' $r_{c}$ '; the correct label is ' $\chi_{r_{c}}$ '. In Figs. If and $3 f$, in all instances of ' $\chi_{s}$ ' subscript ' $S$ ' should have been ' $S_{c}$ '; the correct label is ' $\chi_{S_{c}}$ '. In the $y$-axis labels in Figs. $2 c$ and $4 c$ in ' $P_{s}$ ' subscript ' $s$ ' should have been ' $S$ '; the correct label is ' $P_{s}\left(\delta S_{c}, L\right)$ '. In the $y$-axis labels in Figs. $2 \mathrm{f}$ and $4 \mathrm{f}$ in ' $f_{s}$ ' subscript ' $s$ ' should have been ' $S$ '; the correct label is ' $f_{S}\left(\delta S_{c}, L^{-d_{\mathrm{f} 2}}\right)$ '. In Fig. 2 caption, the two instances of ' $\delta p_{\mathrm{c}}$ ' should have been ' $\delta r_{\mathrm{c}}$ '. In Fig. $5 \mathrm{c}$, the $y$-axis label ' $P_{s}(\delta \Delta)$ ' should have read ' $P_{S}\left(\delta S_{c}\right)$ '. In Fig. 3 caption, 'Analogous to Fig. 1' should have read 'a-f, Analogous to Fig. 1a-f'; in Fig. 4 caption, 'Analogous to Fig. 2' should have read 'a-f, Analogous to Fig. 2a-f'; and in Fig. 5 caption, 'Analogue to Fig. 2a,c' should have read 'a-c, Analogous to Fig. 2a-c'. Furthermore, in Supplementary Figs. 1d, 3d, 5d, 7d, 9d and 11d, on the $y$ axes, ' $\chi_{r}$ ' should have been ' $\chi_{r_{c}}$ '; in Supplementary Figs. $1 \mathrm{f}, 3 \mathrm{f}, 5 \mathrm{f}, 7 \mathrm{f}, 9 \mathrm{f}$ and $11 \mathrm{f}$, on the $y$ axes, ' $\chi_{s}$ ' should have been ' $\chi_{S_{c}}$ '; and in Supplementary Figs. $19 \mathrm{c}$ and $20 \mathrm{c}$, on the $y$ axes, ' $P_{s}(\delta \Delta)$ ' should have been ' $P_{S}\left(\delta S_{c}\right)$ '. All versions of the Article have been amended and the Supplementary Information file replaced.

Published online: 24 February 2020

https://doi.org/10.1038/s41567-020-0837-5

(c) The Author(s), under exclusive licence to Springer Nature Limited 2020 